\title{
Multifocal Large Cystic Liver Metastasis of a Sinonasal Tract Ameloblastoma: An Unusual Clinical Presentation
}

\author{
Xiuli Liu ${ }^{\mathrm{a}, \mathrm{c}}$, Hao Xie ${ }^{\mathrm{a}}$, Huamin Wang ${ }^{\mathrm{b}}$, James McMahon ${ }^{\mathrm{a}}$, Deepa T. Patil ${ }^{\mathrm{a}}$, Lisa M. Yerian ${ }^{\mathrm{a}}$
}

\begin{abstract}
Ameloblastoma is a slowly growing, locally invasive tumor of odontogenic epithelial origin. On rare occasion, a well-differentiated ameloblastoma may metastasize. Recently, we encountered a unique case of metastatic ameloblastoma in the liver in a 48-yearold woman. Preoperative radiographic study revealed multiple cystic lesions in the liver, 6 of which were confirmed intraoperatively. Macroscopic examination of the resected tumors revealed well-circumscribed, encapsulated cystic lesions. Sections from the tumors demonstrated solid interlocking islands with peripheral palisading of "basaloid cells" and squamous differentiation of centrally located cells. The cytology of these tumors was bland and the mitotic activity was low. Immunohistochemistry showed immunoreactivity for CK5/6 and p63, immunoreactivity for CD10 in the peripherally palisading cells and immunoreactivity for CK19 in the centrally located cells. The resected liver cystic tumors were originally diagnosed as "low-grade cystic ameloblastoma-like tumor in the liver" and suggested a possibility of metastasis. The patient was doing well clinically after the resection of her liver lesions. However, CT scan of the head and neck region at another hospital one year later showed an enhancing, expansile, well circumscribed mass in the right nasal cavity. Biopsy and resection of the nasal mass revealed an ameloblastoma invading the maxillary bone. The patient is doing well 2 months after her nasal surgery. To our knowledge, this is the first case of ameloblastoma manifested as liver metastases prior to the identification of a sinonasal primary.
\end{abstract}

Keywords: Liver; Ameloblastoma; CD10; P63; Squamous cell carcinoma; Sinonasal tract

\footnotetext{
Manuscript accepted for publication May 16, 2013

${ }^{\mathrm{a} D e p a r t m e n t ~ o f ~ A n a t o m i c ~ P a t h o l o g y, ~ C l e v e l a n d ~ C l i n i c, ~ C l e v e l a n d, ~}$ Ohio 44195, USA

${ }^{\mathrm{b}}$ Department of Pathology, The University of Texas MD Anderson Cancer Center, Houston, Texas 77030, USA

${ }^{c}$ Corresponding author: Xiuli Liu, Department of Anatomic Pathology, Cleveland Clinic Lerner College of Medicine of Case Western Reserve University, 9500 Euclid Avenue/L25, Cleveland, Ohio 44195, USA. Email: liux3@ccf.org
}

doi: http://dx.doi.org/10.4021/jmc1314w

\section{Introduction}

While epithelial neoplasm with squamous differentiation including conventional squamous cell carcinoma is uncommon in the liver, tumors with discrete basaloid and squamoid features are rare. Some examples include variants of squamous cell carcinoma such as basaloid squamous carcinoma, which shows prominent palisading of basaloid cells and brisk mitotic activity [1]. Several case reports and small series have shown tumors with similar morphology but of low-grade cytology including metastatic ameloblastoma and adamantinoma [2,3]. However, liver metastasis of well-differentiated ameloblastoma generally occurs years after diagnosis of the primary and often follows multiple local recurrences [3].

\section{Case Report}

A 48-year-old woman presented to our hospital with complaints of abdominal distension of greater than 1 year in July 2011. Her past history included an infiltrating ductal adenocarcinoma of the breast diagnosed and resected in 2004. The patient had been doing well after her initial surgery for breast cancer. Physical examination demonstrated marked abdominal distension particularly in the upper quadrants. Laboratory tests revealed normal alkaline phosphatase $(127 \mathrm{U} / \mathrm{L}$ (normal (40-150 U/L)), total bilirubin $(0.4 \mathrm{mg} / \mathrm{dL}$ (normal $0.0-1.5 \mathrm{mg} / \mathrm{dL}$ )), albumin $(4.1 \mathrm{~g} / \mathrm{dL}$ (normal $3.5-5 \mathrm{~g} / \mathrm{dL})$ ), alanine transaminase (ALT) (26 U/L (normal 0 - 45U/L)), aspartate transaminase (AST) (31 U/L (normal 7 - 40U/L)). Imaging studies revealed bilateral multiple liver cysts ranging in size from 4 to $28 \mathrm{~cm}$. The largest cyst $(28 \mathrm{~cm})$ was located in the left lobe. A biopsy (07/27/2011) of the largest cyst was diagnosed as low-grade epithelial neoplasm with squamous differentiation. Upper and lower gastrointestinal endoscopic examination was unremarkable. The patient subsequently developed severe abdominal pain following the initial biopsy and underwent a diagnostic laparoscopy and surgical decompression of the largest cyst with drain placement at the time. Six weeks following the initial biopsy, the patient underwent an exploratory laparotomy for surgical resection 

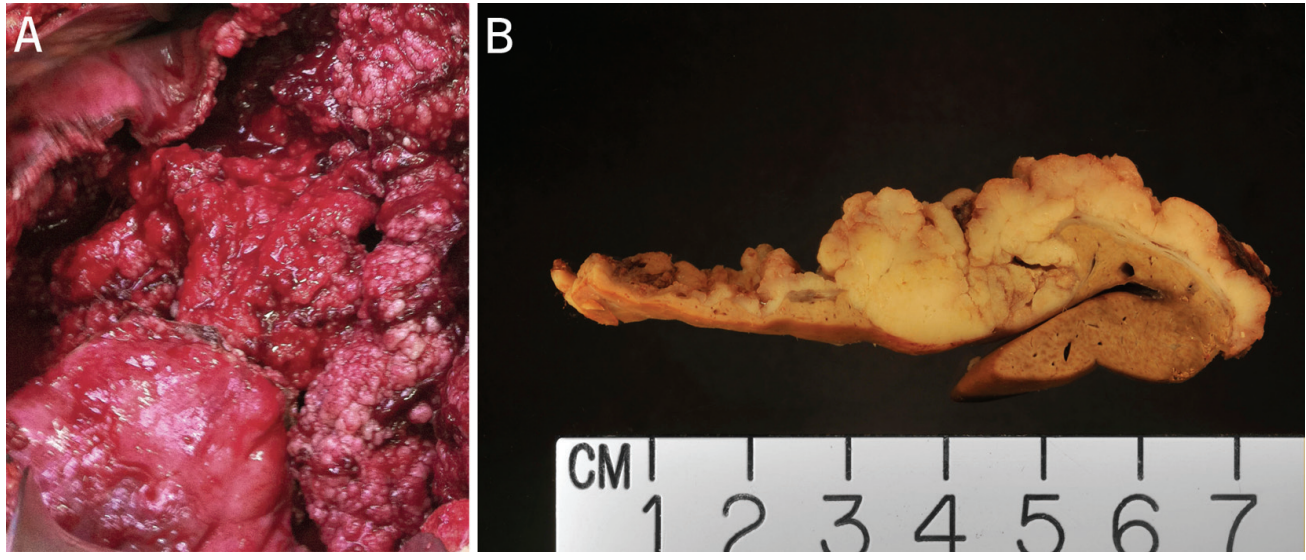

Figure 1. (A) Intraoperative view of the largest cyst. The largest cyst was superficially located and the inner surface of the cyst was irregular with papillae and protuberance of difference sizes and thickness. (B) Cut surface of a portion of the resected cystic lesion from the left lobe of the liver. This cut surface was from the solid area of the cystic lesion. The adjacent cystic wall was irregular with papillation. The tumor was circumscribed, and encapsulated for most part. The adjacent liver did not show evidence of cirrhosis.

of her liver cysts. Because the bilateral, multiple cystic liver lesions and the large size precluded complete resection, fenestration, partial resection and drainage of approximately 4 $\mathrm{L}$ of fluid from the largest cyst were performed and submitted to pathology (parts A and B). No bile was noted in the drained fluid. In addition, a cystic lesion from the left lobe was also resected and submitted to pathology (part C). A total of six cystic lesions were noted in the left and right lobes with partial fenestration performed as was deemed possible.

\section{Macroscopic features of the resected liver cystic lesions}

Parts A and B were from the largest cyst and included two segments of cyst wall which measured $6.5 \times 6 \times 1.1 \mathrm{~cm}$ (part A) and $43 \times 18 \times 2.5 \mathrm{~cm}$ (part B). Overall, this lesion was a large cyst, located in the subcapsular region, for most part, capsulated and had a pushing border. The thickest area of the cyst wall measured $2.5 \mathrm{~cm}$ in the larger piece (part B). The attached liver on the larger piece measured $4.8 \times 4.2 \times$ $0.9 \mathrm{~cm}$ (part B). As observed intraoperatively (Fig. 1A), the inner surface of the cyst was irregular with papillae and protuberance of difference sizes and thickness (parts A \& B, Fig. 1B). Sectioning through the larger piece revealed a small intact cyst which contained clear gelatinous material as well. The adjacent liver does not show gross evidence of cirrhosis (Fig. 1B). The small cystic lesion resected from the left lobe (Part C) had similar macroscopic appearance. Both lesions were extensively sampled for histologic examination.

\section{Material and methods}

Formalin-fixed tissue was processed routinely and stained with H\&E and periodic acid -Schiff (PAS) with diastase digestion. Immunohistochemical stains were performed us- ing the avidin-biotin complex technique, with appropriately positive and negative controls. The following antibodies were used: anticytokeratin (CK5/6 (Milliport/MAB1620 at 1:150 dilution), CK7 (Dako/M7018 at 1:40 dilution), CK19 (Invitrogen/08-0190 at 1:10 dilution)), p63 (Ventana/790-4509 predilute), p53 (Dako/M7001 at 1:20 dilution), p16 (MTM Laboratories/CINtec9517 predilute), ki67 (Ventana/790-4286 predilute), smooth muscle actin (SMA) (Dako/M0851 at 1:50 dilution), CD10 (Novocastra/NCLCD10-270 at 1:5 dilution), S-100 (Dako/Z0311 at 1:200 dilution), and estrogen receptor (Ventana/790-4325 predilute). Tissue for electron microscopy was processed in a routine manner.

Histologic, immunohistochemical, and ultrastructural features of the resected liver cystic lesions

Sections from these two cystic masses showed similar microscopic features. The tumors were cystic and encapsulated in most part (Fig. 2A). The solid component comprises interlocking islands with peripheral palisading of basaloid cells (Fig. 2B). The cells in the periphery of the islands appear basaloid with small hyperchromatic nuclei, no nucleoli, and scant cytoplasm. While most of the peripherally palisading nuclei rest on the basement membrane, focally, reverse polarity is noted for some of the basaloid cells (Fig. 2C). The cells in the central portion of the islands show obvious squamous differentiation with relatively uniform cells containing slightly pleomorphic nuclei, small nucleoli, and moderately abundant eosinophilic cytoplasm (Fig. 2D). Mild cystic degeneration and myxoid changes are noted focally in the central portion of the islands (Fig. 2E), resembling the "stellate reticulum" of the dental enamel origin. Mitotic activity is low with only rare mitotic figures seen. Rare individual tu- 

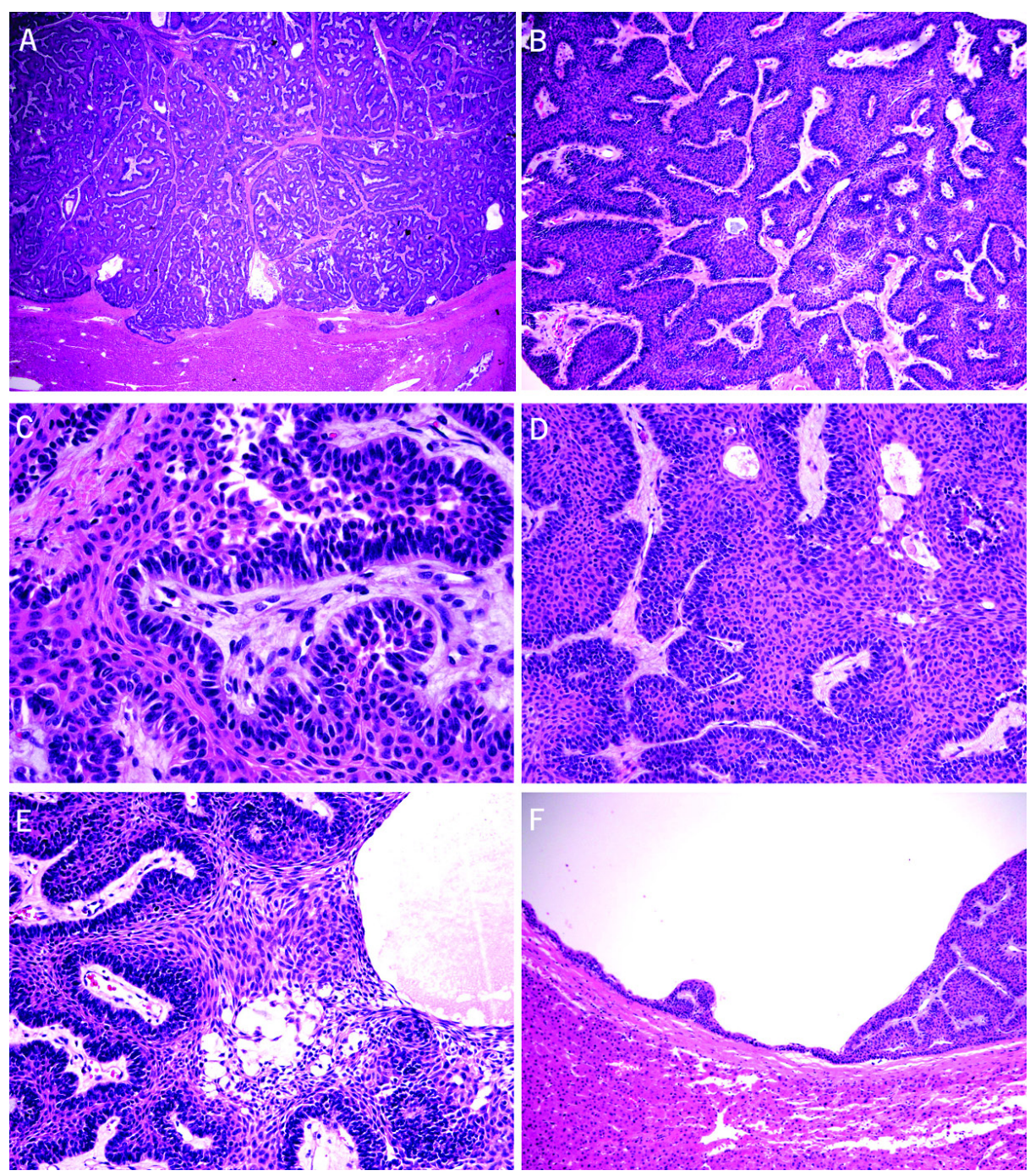

Figure 2. Microscopic features of the resected cystic liver tumors (A-F). (A) Complex architecture of interlocking islands composed of epithelial cells (H\&E stain, 20X). (B) Interlocking islands consisted of peripheral palisading of basaloid cells (H\&E stain, 40X). (C) Focal reverse polarity noted in basaloid cells (H\&E stain, 100X). (D) Obvious squamous differentiation of the centrally located cells with relatively uniform cells containing slightly pleomorphic nuclei, small nucleoli, and moderately abundant eosinophilic cytoplasm (H\&E stain, 100X). (E) Mild cystic degeneration and myxoid changes noted focally in the central portion of the islands, resembling the "stellate reticulum" of the dental enamel origin (H\&E stain, 200X). (F) A few small cysts lined with an epithelial lining with gradual transition from flat epithelium, early papillation, complex papillation, and solid protuberance noted adjacently to the largest cyst (H\&E stain, 20X).

mor cell apoptosis and focal coagulative tumor necrosis are noted, likely related to previous biopsy and decompression procedures. In some areas, papillary structures consisted of myxoid fibrovascular core lined by hyperchromatic basaloid cells were identified. Heterologous elements such as bone, cartilage, fat, skeletal muscle, or neural tissue were not seen. The adjacent liver was atrophic due to compression by the tumor. There was no evidence of cirrhosis in the adjacent liver. A few small cysts containing an epithelial lining with gradual transition from flat epithelium, early papillation, complex papillation, and solid protuberance were noted ad- jacently to the largest cyst (Fig. 2F). The cellular composition in these small cystic lesions was identical to the largest lesion.

Immunohistochemical stains showed that the tumor cells were positive for CK5/6 (Fig. 3A), p63 (Fig. 3B) but negative for CK7, smooth muscle actin (SMA), and S-100 protein. The squamoid cells in the central portion of the islands were positive for CK19 (Fig. 3C). The peripherally palisading cells were strongly positive for CD10 (Fig. 3D). No CD10 positive or estrogen receptor positive cells are noted in the fibrous capsule. Patchy immunoreactivity 


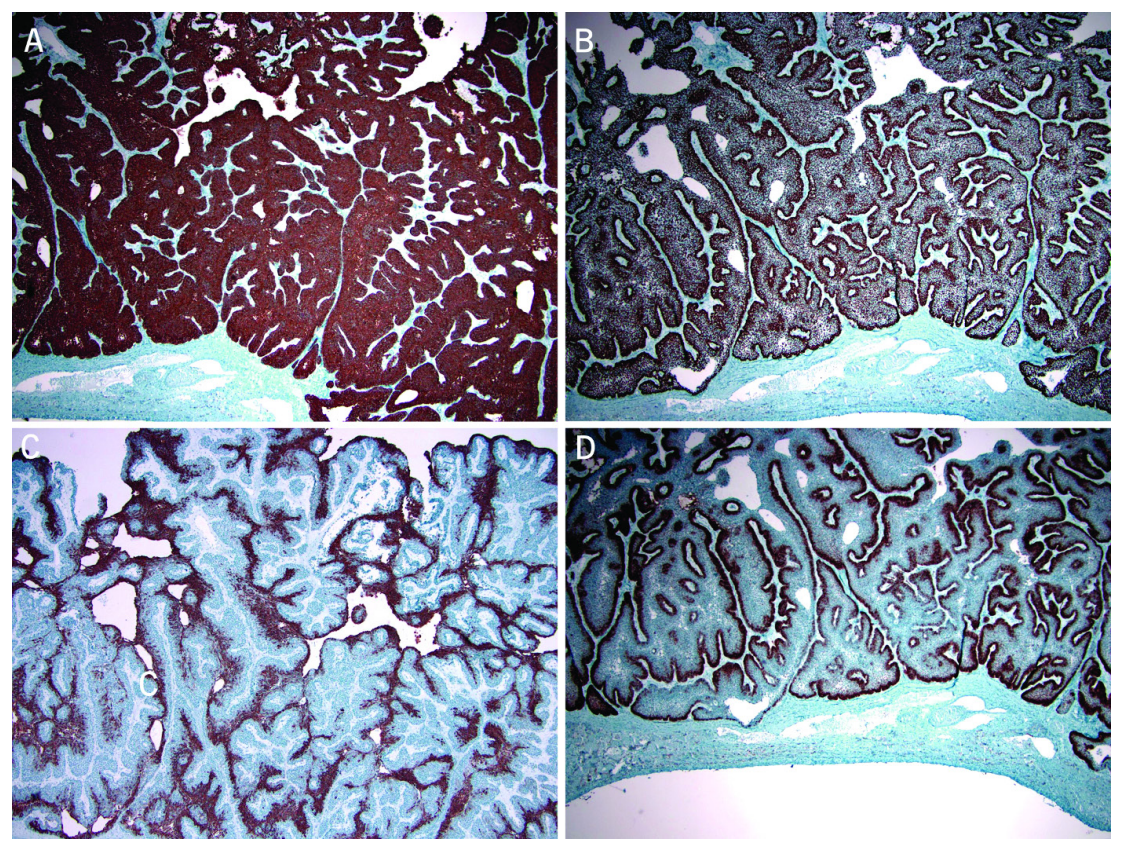

Figure 3. Immunohistochemical stains show the tumor cells were positive for CK5/6 (A, peroxidase stain 40X) and $p 63$ (B, peroxidase stain 40X). The squamoid cells in the central portion of the islands were positive for CK19 $(C$, peroxidase stain $100 X)$. The peripherally palisading cells were strongly positive for CD10 (D, peroxidase stain 40X).

for p53 and p16 were noted in the tumor. Ki67 labeling up to $10-20 \%$ was seen mainly in the peripherally palisading cells. PAS/D stain does not reveal intracellular mucin in tumor cells. Electron microscopy was performed and showed that the "basaloid" cells were invested by a basement membrane at the basal aspect and joined each other by apical junction without clear evidence of tight junction, intermediate junction, or desmosome (micrograph not shown). In the area with histological evidence of squamous differentiation, inter-cellular bridging, desmosome, and abundant tonofilaments were noted; some of the tonofilaments formed perinuclear basket (micrograph not shown). These features were typical of squamous cells. Definite features of normal biliary epithelium or ciliated epithelium were not identified by electron microscopic examination. Based on these findings, the diagnosed of low-grade cystic ameloblastoma-like tumor in the liver was rendered. A possible metastasis was considered but cannot be confirmed at the time of liver resection.

Since both cystic tumors had histology resembling ameloblastoma, a follow-up full body positron emission tomography-computed tomography (PET-CT) scan was performed and revealed a hypermetabolic soft tissue mass in the right inferior nasal cavity and middle and inferior turbinates with adjacent bone remodeling. Otherwise, there was no evidence of lytic or sclerotic bone lesions by PET-CT. The clinical differential diagnosis of this hypermetabolic sinonasal tract soft tissue mass included chronic inflammatory process versus low-grade neoplasm. However, laryngoscopic examina- tion of her sinonasal tract did not reveal lesions and found no definite evidence of a nasopharyngeal neoplasm.

The patient received no chemoradiation following surgery. Three weeks after the surgery, CT scan revealed an interval decrease in her dominant liver masses with no increase in the other cystic lesions. She was doing well and gained approximately 10 pounds with improved energy within 2 months following her surgery. Abdominal CT scan one year later revealed lesions in both lobes of the liver, some of which were cystic and others were more solid in appearance. A large predominantly cystic mass in the right lobe had clearly progressed in size from $8.4 \times 5.7$ to $10.9 \times 7.7 \mathrm{~cm}$, whereas others were either stable or decreased in size (from $8.4 \times 7.2 \mathrm{~cm}$ to $6.6 \times 6.3 \mathrm{~cm}$ ).

One year after the resection of her liver cystic lesions, the patient was referred to another hospital for further workup of her nasal mass. CT scan of the head and neck region showed an enhancing, expansile, well circumscribed mass in the right nasal cavity extending around the right middle and inferior turbinates. The mass invades into the right hard palate, abuts the soft palate and leads to lateral deviation of the medial wall of the right maxillary sinus. The mass extends posteriorly into the ventral aspect of the right nasopharynx. There is no evidence of perineural spread into the pterygopalatine fossa, intracranial spread, cervical adenopathy or involvement of the sphenopalatine foramen. She underwent nasal endoscopy, biopsy of right nasal mass, and infrastructure maxillectomy with septectomy and dental extraction of 

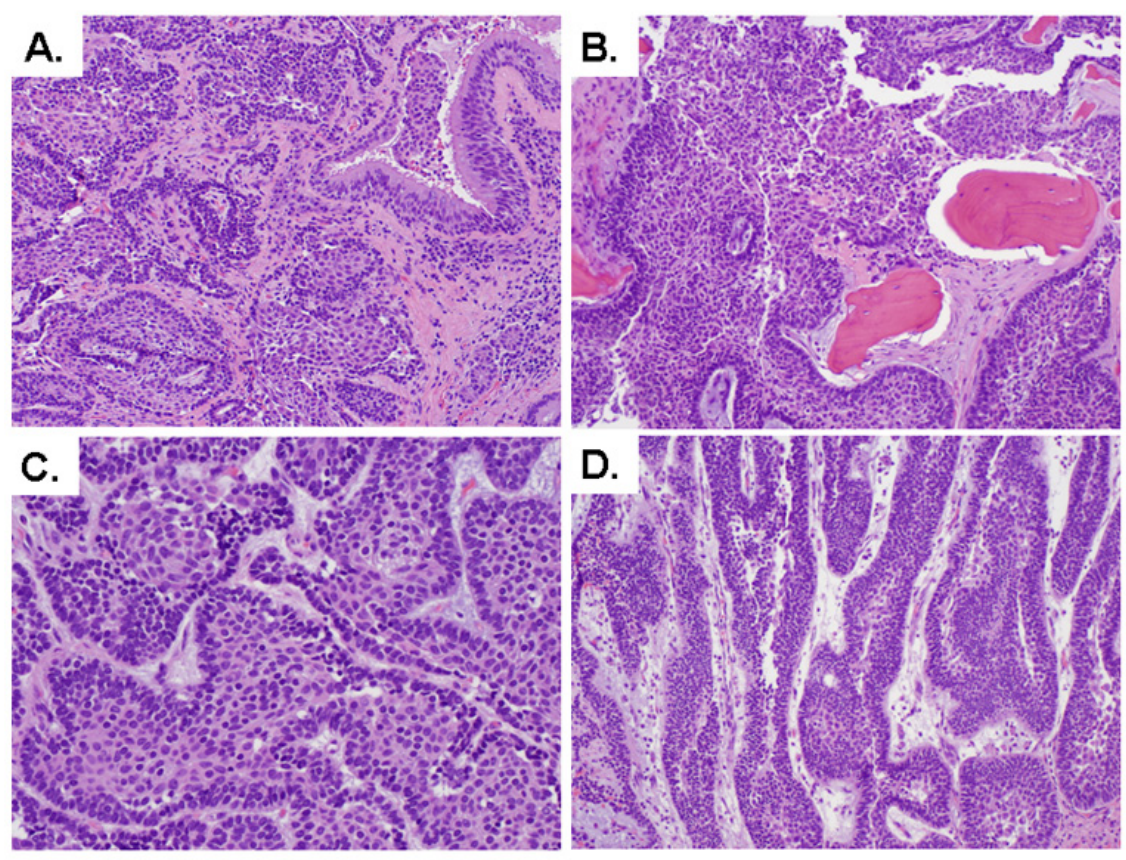

Figure 4. Microscopic features of the resected nasal mass (A-D). (A) Tumor invades nasal mucosa (H\&E stain, 100X). (B) Tumor permeates maxillary bone (H\&E stain, 100X). (C) Interlocking islands consisted of peripheral palisading of basaloid cells (H\&E stain, 400X). (D) Focal plexiform configuration (H\&E stain, 200X).

right upper incisor. In the posterior aspect along the floor and lateral wall of the nasal cavity, there is an exophytic friable mass $(2.5 \times 1.7 \times 1.1 \mathrm{~cm})$ extends close to posterior soft tissue margin with other edges widely free. The mucosa anterior to the tumor has vague nodularity $(0.7 \mathrm{~cm}$ in greatest dimension). Microscopic examination of the right infrastructure maxillectomy showed that the tumor focally invaded nasal mucosa (Fig. 4A) and permeated the maxillary bone (Fig. 4B). A predominant of follicular pattern (Fig. 4C) and a focal plexiform configuration (Fig. 4D) are present, which is morphologically identical to the liver tumors. The morphology of the liver lesions and the nasal mass was different from the patient's infiltrative ductal carcinoma of the breast (data not shown). Therefore, the final diagnosis of metastatic ameloblastoma to the liver was rendered.

\section{Discussion}

Given the tumor multiplicity in the liver, metastatic ameloblastoma was strongly considered at the time of liver resection. This diagnosis is extraordinarily rare in the liver given the absence of a known history of an odontogenic ameloblastoma. It is known that ameloblastomas may arise in the sinonasal tract [4] but none of cases in the reported literature experienced metastases. Our patient was noted to have a right inferior nasal cavity mass on PET-CT at the time when her liver masses were identified. However, no lesions were iden- tified on endoscopic examination of her sinonasal tract. In addition, the tumors were confined to her liver and no lesions were identified in other sites during the follow-up period. A tumor that is histologically similar to the ameloblastoma is adamantinoma. This tumor is almost exclusively found in the tibia. Rare examples of metastatic ameloblastoma and adamantinoma have been reported in the liver [2, 3]. However, no bone lesions were identified in our patient during her follow-up PET-CT. In addition, esophagogastroduodenoscopic and colonoscopic examination revealed normal gastrointestinal tract making a metastatic basaloid squamous cell carcinoma from the gastrointestinal tract unlikely.

Since the patient had a clinical history of breast cancer, a possibility of metastasis from the breast cancer should also be considered as metastasizing and relapsing "low grade" adenosquamous metaplastic breast cancer have been reported [5]. However, thorough histologic review of the mastectomy specimen in our case revealed a poorly differential ductal carcinoma with medullary features; no metaplastic component such as squamous differentiation was noted. There was no histologic resemblance between her breast cancer and her liver tumors.

Other potential differential diagnoses for the unique histology of the resected liver cystic tumors include a lowgrade counterpart of basaloid squamous cell carcinoma, a biliary papilloma with complete squamous metaplasia with a mixed endophytic and exophytic/inverted growth pattern, or a low-grade ameloblastoma-like tumor arising from pre- 
existing congenital/foregut cysts. Regarding its exact origin, we can only speculate at the time of her initial liver tumor resection as no primary has been found. Extensive sampling of the tumors did not show a single focus of normal biliary epithelium and the lack of communication to the biliary duct cyst (no bile was noted in the drained cystic fluid), making the possibility of inverted biliary adenoma unlikely. Further, the tumor cells did not show immunoreactivity for CK7, a sensitive, but not specific marker of biliary epithelium, further refuting the possibility of inverted biliary adenoma. Other possibilities include a neoplastic transformation of a pre-existing cyst lined by epithelium with squamous metaplasia such as congenital cysts including foregut cysts or cystic teratoma. Indeed, we observed a gradual transition from flat "metaplastic" epithelium, early papillation, protuberance, and finally solid area in some small cysts adjacent to the largest cyst. However, neither ciliated epithelium nor heterologous elements such as bone, cartilage, fat, skeletal muscle, or neural tissue were identified in multiple sections from these two tumors.

Our patient had close postoperative followup by imaging study. CT examination of her abdomen one year after her liver surgery revealed lesions in both lobes of the liver, some of which were cystic and others were more solid in appearance. A large predominantly cystic mass in the right lobe had clearly progressed in size from $8.4 \times 5.7 \mathrm{~cm}$ to 10.9 $\times 7.7 \mathrm{~cm}$, whereas others were either stable or decreased in size from $8.4 \times 7.2 \mathrm{~cm}$ to $6.6 \times 6.3 \mathrm{~cm}$. Further workup of her nasal cavity mass at another hospital one year after the resection of her liver cystic lesions, by imaging, biopsy and resection identified a sinonasal ameloblastoma as a primary. The patient was doing well 2 months after her nasal surgery.

Ameloblastoma is a slowly growing, locally invasive tumor of odontogenic epithelial origin. On rare occasion, a well-differentiated ameloblastoma may give rise to nodal or hematogenous metastasis [3]. Hematogenous metastasis usually follows multiple failed attempts at primary tumor control, occurs with an average time of 18 years ( 3 - 45 years) after the diagnosis of primary, and most commonly involves the lung [3]. Liver metastasis of ameloblastoma is extremely rare and the reported few cases occurred years after the diagnosis of primary and usually followed multiple local recurrence [3]. Most metastatic ameloblastomas reported in the literature had an origin from either mandibular or maxillary bone [3]. In the current case, the primary ameloblastoma of the sinonasal tract was diagnosed 1 year after the resection of the liver cystic lesions of unusually large size and retrospectively confirmed the liver low-grade cystic epithelial neoplasms as metastatic ameloblastoma. Treatment for metastatic ameloblastoma varies but includes surgical resection, chemotherapy, radiation, or a close followup/watch approach [3]. In one report, over half of the patients were alive and had survived an average of 10 years since diagnosis of metastasis [3]. For those patients who had succumbed to their disease had an average survival time of 2.6 to 3 years after diagnosis of metastasis $[3,6]$. Our current patient was doing well 14 months after diagnosis of metastasis.

In summary, we report an extremely rare case of metastatic ameloblastoma in the liver from a sinonasal tract primary which was only diagnosed 1 year after the initial resection of the liver metastatic tumors. Our current report strongly advocates that close clinical followup is needed to identify the primary in patients with multifocal, cystic, lowgrade epithelial neoplasm with morphology resembling ameloblastoma.

\section{Grand Support}

None.

\section{Conflict of Interest}

None declared.

\section{References}

1. De Boer WB. Basaloid squamous cell carcinoma in the liver. Pathology. 2000;32:147-151.

2. Beppu H, Yamaguchi H, Yoshimura N, Atarashi K, Tsukimoto K, Nagashima Y. Adamantinoma of the rib metastasizing to the liver. Intern Med. 1994;33(7):441445.

3. Van Dam SD, Unni KK, Keller EE. Metastasizing (malignant) ameloblastoma: review of a unique histopathologic entity and report of Mayo Clinic experience. J Oral Maxillofac Surg. 2010;68(12):2962-2974.

4. Schafer DR, Thompson LD, Smith BC, Wenig BM. Primary ameloblastoma of the sinonasal tract: a clinicopathologic study of 24 cases. Cancer. 1998;82(4):667674.

5. Kinkor Z, Skalova A, Michal M, Janousek M, Kheck M. [Metastasing and relapsing "low grade" adenosquamous metaplastic breast cancer--is there a really indolent lesion? A description of three cases and review of literature]. Ceska Gynekol. 2005;70(3):211-216.

6. Kunze E, Donath K, Luhr HG, Engelhardt W, De Vivie R. Biology of metastasizing ameloblastoma. Pathol Res Pract. 1985;180(5):526-535. 